\title{
Calcium-dependent action potentials in mouse spinal cord neurons in cell culture
}

\author{
ERIC J. HEYER, ROBERT L. MACDONALD*, GREGORY K. BERGEY and PHILLIP G.
} NELSON

Department of Neurology, University of Michigan Medical Center, Ann Arbor, Mich., and (G.K.B. and P.G.N.) Laboratory of Developmental Neurobiology, National Institutes of Health, Bethesda, $M d$. (U.S.A.)

(Accepted May 7th, 1981)

Key words: calcium - - action potential - spinal cord neurons - cell culture

Following blockade of membrane potassium conductance with tetraethylammonium ions or 3aminopyridine, long-duration action potentials were recorded from mouse spinal cord neurons in primary dissociated cell culture. The action potentials were calcium-dependent since they: (1) were not blocked by the sodium-channel blocker tetrodotoxin, (2) could be recorded in sodium-free, calciumcontaining medium (3) could not be evoked in sodium-containing, calcium-free medium, (4) were blocked by calcium channel blockers manganese and cobalt and (5) had overshoot amplitudes that varied linearly with the log of the extracellular calcium concentration (slope of $27.5 \mathrm{mV} /$ decade change in calcium concentration).

Mouse spinal cord and dorsal root ganglion neurons in primary dissociated cell culture have excitable membrane properties ${ }^{31,32}$. Action potentials recorded from dorsal root ganglion neurons in cell culture have both sodium and calcium components ${ }^{31}$; however, action potentials recorded from spinal cord neurons in cell culture are only sodium-dependent since action potentials could not be evoked when the neurons were bathed in sodium-free medium or following addition of the sodium channel blocker tetrodotoxin (TTX) to sodium-containing bathing medium ${ }^{31}$. This result, however, did not exclude the presence of a voltage-dependent calcium conductance in spinal cord neurons. In fact, while action potentials in squid axon were sodium-dependent, two phases of calcium entry into squid axons followed membrane depolarization ${ }^{3}$. The first was blocked by TTX and thus probably represented calcium movement through sodium-selective channels. The second phase was blocked by manganese and thus represented calcium movement through calcium-selective channels. This late increase in calcium conductance, however, was insufficient to overcome the large increase in repolarizing voltage-dependent potassium conductance ${ }^{14}$, and thus despite the presence of a voltage-dependent calcium conductance, depolarization

*Send correspondence to: Dr. Robert L. Macdonald, 1103 East Huron, Ann Arbor, Mich. 48109, U.S.A. 
of squid axons produced only sodium-dependent action potentials. In many other neurons, however, calcium-dependent action potentials have been recorded from all portions of neuronal membranes including dendrites ${ }^{22,24,33,37}$, cell bodies $1,7,9,11,21$, ${ }^{25-28}$, axons ${ }^{15}$ and synaptic terminals ${ }^{20,23}$ and have been recorded from a variety of different cell types including vertebrate central $22,24,33,37$ and peripheral ${ }^{7,25-27,38}$ nervous system neurons, invertebrate neurons ${ }^{9,11,21}$ and neuroblastoma cells ${ }^{28}$. In other neurons which appeared to have only sodium-dependent action potentials, reduction of the voltage-dependent potassium conductance by either tetraethylammonium ion (TEA) or 3-aminopyridine (3-AP) has permitted recording of calcium-dependent action potentials $4,15,27,29$. In this communication we will demonstrate that longduration action potentials could be recorded from mouse spinal cord neurons in cell culture following addition of TEA, and that these action potentials were calciumdependent.

Cell cultures were prepared from spinal cords and attached dorsal root ganglia dissected from 12-13.5 day old fetal mice as previously described ${ }^{32}$. Following trypsinization and mechanical disruption of the tissue, the resultant cell suspension was plated on collagen-coated $35-\mathrm{mm}$ culture dishes such that 2-4 cultures were obtained for each spinal cord. Cultures were maintained at $35^{\circ} \mathrm{C}$ in $10 \%$ carbon dioxide and $90 \%$ room air in a growth medium containing Eagle's minimal essential medium and $10 \%$ horse serum. Electrophysiological experiments were performed 4-12 weeks following plating.

Intracellular recordings were made with glass micropipettes (25-50 M $\Omega$ ) filled with $4 \mathrm{M}$ potassium acetate. A conventional bridge circuit permitted simultaneous measurement of membrane potential and injection of current through a single micropipette. Maximum rates of rise $\left(\dot{\mathrm{V}}_{\max }\right)$, duration and overshoot magnitudes were measured from film of action potentials obtained from the screen of a storage oscilloscope.

Unless otherwise noted, recordings were made at $35-37{ }^{\circ} \mathrm{C}$ in a high calciumbalanced salt solution (control bathing solution) consisting of (in $\mathrm{mM}$ ): $\mathrm{NaCl}$ 137.5; $\mathrm{KCl} 5.3 ; \mathrm{CaCl}_{2} 5.0 ; \mathrm{MgCl}_{2}$ 0.8; Tris- $\mathrm{HCl} 13$; and glucose 5.6. Osmolarity was 300-310 mOsm and $\mathrm{pH}$ was 7.2-7.4. Calcium concentrations were varied by mixing isosmotic stock solutions of 1-20 mM calcium chloride (sodium chloride concentration was varied to maintain osmolarity). We did not use calcium chelators and thus calcium-free solutions probably contained $20-100 \mu \mathrm{M}_{\text {calcium }}{ }^{10}$. When TEA (chloride or bromide) (15-100 mM) or 3-AP (5 mM) was added, sodium chloride concentration was lowered to maintain constant osmolarity. In sodium-free solutions, choline or Tris was substituted for sodium. TTX was added from a $1 \mathrm{mM}$ stock solution to obtain concentrations of 1-3 $\mu \mathrm{M}$. Calcium and manganese ion concentration were altered locally around individual neurons by pressure ejecting the bathing solution containing the desired divalent ion concentration from micropipettes whose tips were broken to tip diameters of 2-5 $\mu \mathrm{m}$. Controls for pressure artifact were always obtained using pipettes filled with control medium.

Depolarization of spinal cord neurons with brief $(0.4 \mathrm{~ms})$ currents evoked action potentials with short duration $(0.6 \mathrm{~ms})$ and high $\dot{\mathrm{V}}_{\max }(243.8 \mathrm{~V} / \mathrm{s})$ (Table I; Fig. $1 \mathrm{~A}_{1}$, 
TABLE I

Action potential properties of spinal cord neurons

Each entry is the mean \pm standard error of the mean. The number of neurons used for each entry is shown in brackets following each entry. Concentration of TEA was $100 \mathrm{mM}$ and of TTX was $1 \mu \mathrm{m}$.

\begin{tabular}{lcc}
\hline & Duration $(\mathrm{msec})$ & $\dot{V}_{\max }(\mathrm{V} / \mathrm{s})$ \\
\hline Control & $0.6 \pm 0.2(11)$ & $243.8 \pm 35.9(10)$ \\
Calcium-free & $1.0 \pm 0.2(6)^{* * *}$ & $204.4 \pm 27.8(8)$ \\
Sodium-free + TTX + TEA & $678.6 \pm 305.3(5)^{* *}$ & $30.2 \pm 9.6(12)^{*}$ \\
\hline
\end{tabular}

* Significant at the 0.001 level using Student's $t$-test.

** Significant at the 0.01 level, *** Significant at the 0.05 level.

$\left.B_{1}\right)$. Action potentials could not be evoked, however, following addition of TTX to the bathing medium ( 79 cells) or in sodium-free medium (12 cells). In calcium-free medium, action potentials were slightly longer $(1.0 \mathrm{~ms})$, but $\dot{\mathrm{V}}_{\max }(204.4 \mathrm{~V} / \mathrm{s})$ was unaltered (Table I; Fig. 1A2). With addition of $100 \mathrm{mM}$ TEA to sodium-free, TTXcontaining bathing medium, however, depolarizing stimulation elicited slow regenerative responses of long duration $(678.6 \mathrm{~ms})$ and low $\dot{\mathrm{V}}_{\max }(30.2 \mathrm{~V} / \mathrm{s})$ (Table I; Fig. $1 \mathrm{~B}_{2}$ ). The long-duration action potentials could be evoked in almost all neurons if sufficient current could be passed to depolarize the neuronal membrane by $20-30 \mathrm{mV}$ for 10-20 s. Action potential duration in TTX- and TEA-containing medium was dependent on both membrane potential and frequency of stimulation. Membrane hyperpolarization reduced and membrane depolarization increased action potential duration. Short interstimulus intervals (usually $<10 \mathrm{~s}$ ) reduced action potential duration. Therefore, in all experiments, we allowed the membrane potential to stabilize before making duration measurements and stimulated with large interstimulus intervals (usually $30 \mathrm{~s}$ ).

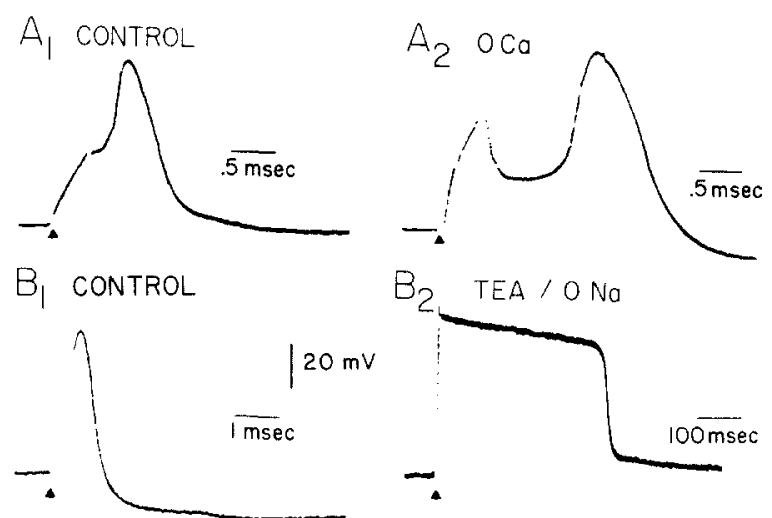

Fig. 1. Sodium- and calcium-dependent action potentials elicited from spinal cord neurons in cell culture. Action potentials were elicited in spinal cord neurons at resting membrane potential (RMP) by applying brief depolarizing currents through the recording micropipettes using the bridge technique; stimulus onset in this and subsequent figures are denoted by filled triangles. Cultures were bathed in control medium $\left(A_{1}, B_{1}\right)$, calcium-free medium $\left(A_{2}\right)$ or sodium-free medium containing 100 $\mathrm{mM}$ TEA and $1 \mu \mathrm{M}$ TTX $\left(B_{2}\right)$. Stimulus durations were $0.4 \mathrm{msec}$ in $A_{1}$ and $A_{2}, 0.1 \mathrm{msec}$ in $B_{1}$ and 10 msec in $B_{2}$. RMP was $-62 \mathrm{mV}$ in $A_{1},-52 \mathrm{mV}$ in $A_{2},-56 \mathrm{mV}$ in $B_{1}$ and $-63 \mathrm{mV}$ in $B_{2}$. These and subsequent tracings were retouched to remove grid markings. 


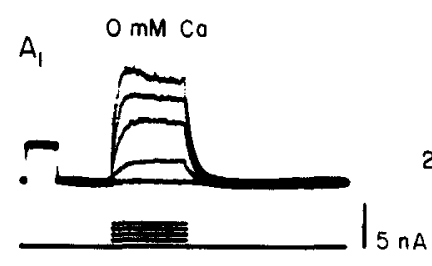

$\mathrm{B}_{\mathrm{I}} 6 \mathrm{mMCa}+6 \mathrm{mMCo}$

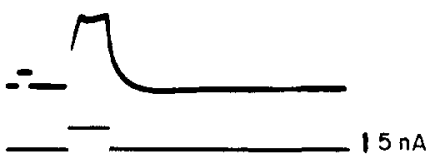

$6 \mathrm{mM} \mathrm{Ca}$

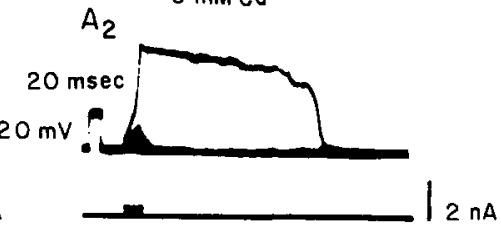

$\mathrm{B}_{2}$

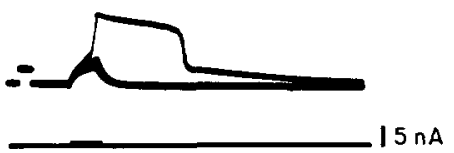

Fig. 2. Long-duration action potentials recorded in TEA- and TTX-containing medium were calciumdependent and blocked by cobalt. Depolarizing stimuli were applied to spinal cord neurons bathed in HEPES-buffered saline containing $1 \mu \mathrm{M}$ TTX and $25 \mathrm{mM}$ TEA with varying calcium (Ca) and cobalt (Co) concentrations. All calibration pulses were $20 \mathrm{mV} \times 20 \mathrm{~ms}$.

The long-duration action potentials evoked in TTX- and TEA-containing medium were calcium-dependent. Depolarization of spinal cord neurons bathed in calcium-free medium evoked no regenerative responses (Fig. 2 $\mathrm{A}_{1}$ ) in 12 neurons. In contrast, smaller stimulating currents evoked long-duration regenerative responses in medium containing $6 \mathrm{mM}$ calcium (Fig. 2A 2 ). Cobalt is a calcium channel blocker and no regenerative responses could be evoked in 13 neurons bathed in calcium- and cobalt-containing medium (Fig. 2B $\mathrm{B}_{1}$ ). However, upon washing the culture and replacing the medium with a cobalt-free medium, long-duration regenerative responses could again be evoked ( 4 out of 5 neurons).

The calcium dependency of the long duration action potentials could also be demonstrated on single neurons by pressure application of calcium- or manganesecontaining solutions. Membrane depolarization of neurons in calcium-free bathing medium containing TTX, TEA and 3-AP did not evoke regenerative responses (Fig.

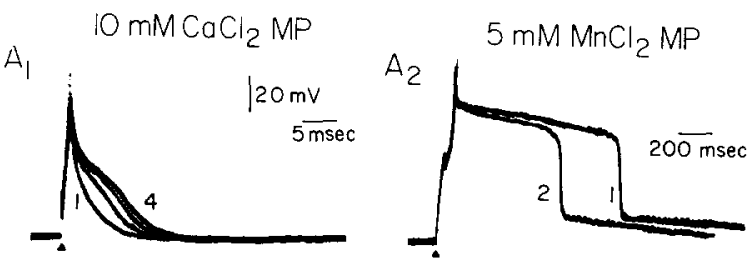

Fig. 3. Calcium increased and manganese reduced action potential duration. Action potentials were evoked in calcium-free $\left(A_{1}\right)$ or control $\left(A_{2}\right)$ bathing medium containing $15 \mathrm{mM}$ TEA, $5 \mathrm{mM}$ 3-AP and $3 \mu \mathrm{M}$ TTX. A 1 : application of $1 \mathrm{~ms}$, depolarizing stimuli did not evoke action potentials (trace 1 ). After a miniperfusion pipette containing the same bathing medium but with $10 \mathrm{mM} \mathrm{CaCl}$ added was positioned next to the cell and medium was ejected, a long-duration action potential was elicited (trace 4). Following removal of the calcium-containing pipette, the evoked action potentials become shorter, and after a few stimuli, no action potential was elicitable. $\mathbf{A}_{\mathbf{2}}$ : action potential (trace 1) duration and plateau amplitude were reduced $15 \mathrm{~s}$ after a 4-s application of bathing solution containing $5 \mathrm{mM}$ manganese ion (trace 2). Following removal of the manganese-containing miniperfusion pipette, action potential duration on overshoot returned to control values. RMPs were $-54 \mathrm{mV}$ in $\mathrm{A}_{1}$ and $-61 \mathrm{mV}$ in $\mathrm{A}_{2}$. 
$3 \mathrm{~A}_{1}$, trace 1). However, after application of calcium-containing medium from a miniperfusion pipette, long-duration action potentials could be evoked (Fig. $3 \mathrm{~A}_{1}$, trace 4). After removal of the calcium-containing pipette, no regenerative responses could be evoked. The long-duration action potentials were also blocked by the application of the calcium channel blocker manganese. Action potential plateau amplitude and duration were reversibly decreased when bathing medium containing $5 \mathrm{mM}$ manganese ion was pressure ejected from a miniperfusion pipette (Fig. 3A ).

Action potential overshoot was dependent upon extracellular calcium concentration. For example, increase of the calcium concentration of the bathing medium from $5 \mathrm{mM}$ to $20 \mathrm{mM}$ increased the maximum action potential overshoot from +8 $\mathrm{mV}$ to $+28 \mathrm{mV}$ (Fig. 4, right). Maximum action potential overshoot varied linearly with the $\log _{10}$ of the calcium concentration of the bathing medium with a slope of 27.5 $\mathrm{mV}$ for a tenfold change in calcium concentration (Fig. 4, left).

Thus, while action potentials of spinal cord neurons were sodium-dependent and TTX-sensitive in control bathing medium, long-duration calcium-dependent action potentials could be evoked if potassium conductance was reduced by TEA and/or 3AP and sodium conductance was blocked by TTX. These action potentials were calcium-dependent since they: (1) were not blocked by TTX, (2) could be evoked in sodium-free, calcium-containing medium, (3) could not be evoked in sodium-containing, calcium-free medium, (4) were blocked by manganese and cobalt and (5) had overshoot amplitudes that varied linearly with the $\log$ of the extracellular calcium concentration with a slope $(27.5 \mathrm{mV} /$ decade change in calcium concentration) which was close to the theoretical Nernst potential slope for a divalent cation at $35^{\circ}(30.8$ $\mathrm{mV} /$ decade change in calcium concentration).

Mixed sodium-calcium action potentials have been recorded in mouse $25,26,31,38$ and chick $^{7}$ dorsal root ganglion neurons in cell culture, but only sodium-dependent

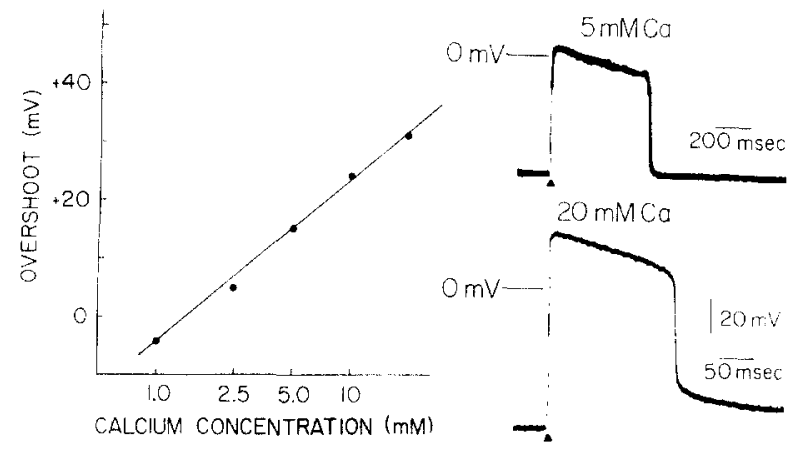

Fig. 4. Action potential overshoot varied as a function of extracellular calcium concentration. Action potentials were elicited from spinal cord neurons in bathing solutions containing $3 \mu \mathrm{M}$ TTX, $100 \mathrm{mM}$ TEA and different calcium concentrations. Action potential overshoot increased when calcium concentration was increased from $5 \mathrm{mM}$ to $20 \mathrm{mM}$ (right). RMP was $-64 \mathrm{mV}$ (top) and $-74 \mathrm{mV}$ (bottom). Average action potential overshoot was linearly related to the $\log _{10}$ of the extracellular calcium concentration (left). Each point represents the average overshoot amplitude of 3-16 neurons except the point at $1.0 \mathrm{mM}$ where only one cell was used (due to the difficulty of evoking calcium action potentials at $1.0 \mathrm{mM}$ ). Standard error of the mean was less than $\pm 15 \%$ of the mean overshoot for each calcium concentration. 
action potentials have been recorded in mouse spinal cord neurons in cell culture ${ }^{31}$. However, our demonstration of long-duration calcium-dependent action potentials following addition of TEA and TTX required a voltage-dependent membrane calcium conductance. It could be that calcium-dependent action potentials are generated in vivo at dendritic sites with either a high density of voltage-dependent calcium channels or a low density of voltage dependent potassium channels. Such dendritic calciumdependent action potentials have been demonstrated in $\mathrm{CA}_{1}$ and $\mathrm{CA}_{3}$ hippocampal pyramidal neurons ${ }^{33,37}$ and cerebellar Purkinje neurons ${ }^{22,24}$. In spinal cord neurons, however, only sodium-dependent action potentials have been recorded unless noncalcium conductances were reduced by specific blocking compounds. Following addition of TEA to the isolated frog spinal cord, slow regenerative depolarizing responses could be evoked in anterior horn cells by depolarizing stimuli ${ }^{4}$. These slow action potentials could not be evoked in low-calcium solutions and were blocked by the calcium channel blocker manganese. Thus under special conditions it has been shown that calcium-dependent action potentials could be evoked in vivo in spinal cord anterior horn cells.

The induction of calcium-dependent action potentials may have important implications for the pathogenesis of seizures and epilepsy. In slices of guinea pig hippocampus, the convulsant penicillin (PCN) induced paroxysmal activity that resembled dendritically generated calcium-dependent action potentials ${ }^{36}$. It was concluded that PCN did not directly alter calcium conductance, but rather, that PCN reduced GABAergic inhibitory postsynaptic potentials so that excitatory postsynaptic potentials could depolarize the dendritic membrane to the calcium conductance threshold and thus produce calcium-dependent action potentials. In cat spinal cord anterior horn cells, PCN application produced paroxysmal bursting ${ }^{18,19}$ and induced a slow persistent inward current which was thought to be a calcium current. The inward current was probably responsible for producing bursting in these neurons ${ }^{34}$. We have demonstrated that in addition to antagonizing GABA-mediated postsynaptic inhibition, PCN and the convulsant bicuculline directly augmented calcium-dependent action potentials in mouse spinal cord neurons ${ }^{12}$. Thus, convulsants have a non-synaptic action on membrane properties as well as a synaptic action to reduce inhibition and both may be involved in their mechanism of action.

The demonstration of calcium-dependent action potentials in spinal cord neurons may be of importance for the study of synaptic transmission. Synaptic transmission is a calcium-dependent process, and it has been shown that presynaptic depolarization produces a voltage-dependent increase in calcium conductance ${ }^{20,23}$. The calcium entry which results is essential for depolarization-secretion coupling in presynaptic terminals. Control of this calcium conductance may thus be essential in regulation of synaptic transmission. For example, the neurotransmitters GABA, norepinephrine, 5-hydroxytryptamine and enkephalin have been shown to decrease the duration of calcium-dependent action potentials of chick dorsal root ganglion neurons in primary dissociated cell culture ${ }^{8,29}$ and norepinephrine inhibited calcium-dependent potentials in rat sympathetic neurons ${ }^{16}$. The results suggest that these neurotransmitters may produce inhibition of synaptic transmission by reduction 
of presynaptic calcium entry. Furthermore, we have demonstrated that the pharmacologically active barbiturates pentobarbital and phenobarbital but not the inactive barbiturate barbituric acid reduce calcium-dependent action potential duration of mouse spinal cord neurons in cell culture at anesthetic concentrations ${ }^{13}$. Barbiturates have been demonstrated to decrease calcium-accumulation by cortical synaptosomes $^{5}$ and to reduce transmitter release ${ }^{6,17,35}$. Our observations suggest that barbiturates produce anesthesia, at least in part, by reducing presynaptic calcium entry, thereby blocking synaptic transmission. Thus, while presynaptic terminals cannot be studied directly, somatic calcium-dependent action potentials of spinal cord neurons in cell culture may provide a model for calcium entry into presynaptic terminals.

We would like to thank Mr. Frank Wilk for technical assistance and Ms. Kathy Lundquist for secretarial assistance.

This study was supported by NIH grant 1 RO1NS15225 (R.L.M.), in part by the Grass Foundation Morison Fellowship (E.J.H.), and part by the NIH Research Career Development Award 1KO4NS00408 (R.L.M.).

1 Adams, D. J. and Gage, P. W., Ionic currents in response to membrane depolarization in an Aplysia neurone, J. Physiol. (Lond.), 289 (1979) 115-141.

2 Adams, D. J. and Gage, P. W., Characteristics of sodium and calcium conductance changes produced by membrane depolarization in an Aplysia neurone, J. Physiol. (Lond.), 289 (1979) 143-161.

3 Baker, P. F., Hodgkin, A. L. and Ridgway, E. B., Depolarization and calcium entry in squid giant axons, J. Physiol. (Lond.), 218 (1971) 709-755.

4 Barrett, E.F. and Barrett, J. N., Separation of two voltage-sensitive potassium currents and demonstration of a tetrodotoxin-resistant calcium current in frog motoneurones, J. Physiol. (Lond.), 255 (1976) 737-774.

5 Blaustein, M. P. and Ector, A. C., Barbiturate inhibition of calcium uptake by depolarized nerve terminals in vitro, Molec. Pharmacol., 11 (1975) 369-378.

6 Cutler, R. W. P. and Young, J., Effect of barbiturates on release of endogenous amino acids from rats cortex slices, Neurochem. Res., 4 (1979) 319-329.

7 Dichter, M. A. and Fischbach, G. D., The action potential of chick dorsal root ganglion neurones maintained in cell culture, J. Physiol. (Lond.), 267 (1977) 281-298.

8 Dunlop, K. and Fischbach, G. D., Neurotransmitters decrease the calcium component of sensory neurone action potentials, Nature (Lond.), 276 (1978) 837-839.

9 Eckert, R. and Lux, H. D., A voltage-sensitive persistent calcium conductance in neuronal somata of Helix pomatia, J. Physiol. (Lond.), 254 (1976) 129-151.

10 Frankenhacuser, B. and Hodgkin, A. L., The action of calcium on the electrical properties of squid axons, J. Physiol. (Lond.), 137 (1957) 218-244.

11 Gelduldig, D. and Junge, D., Sodium and calcium components of action potentials in the Aplysia giant neurone, J. Physiol. (Lond.), 199 (1968) 342-365.

12 Heyer, E. J. and Macdonald, R. L., Membrane depolarization and prolongation of calcium-dependent action potentials of mouse neurons in cell culture by two convulsants: bicuculline and penicillin, Brain Research, in press.

13 Heyer, E. J. and Macdonald, R. L., Barbiturates reduce the duration of calcium-dependent action potentials of mouse spinal cord neurons in primary dissociated cell culture, Neurology, 30(1981) 157

14 Hodgkin, A. L. and Huxley, A. F., A quantitative description of membrane current and its application to conduction and excitation in nerve, J. Physiol. (Lond.), 117 (1952) 500-544.

15 Horn, R., Propagating calcium spikes in an axon of Aplysia, J. Physiol. (Lond.), 281 (1978) 513-534.

16 Horn, J. P. and McAfee, D. A., Norepinephrine inhibits calcium-dependent potentials in rat sympathetic neurons, Science, 204 (1979) 1233-1235. 
17 Kalant, H. and Grose, W., Effects of ethanol and pentobarbital on release of acetylcholine from cerebral cortex slices, J. pharmacol. exp. Ther., 158 (1967) 386-393.

$18 \mathrm{Kao}, \mathrm{L}$. I. and Crill, W. E., Penicillin-induced segmental myoclonus. I. Motor responses and intracellular recording from motoneurons, Arch. Neurol., 26 (1972) 156-161.

19 Kao, L. I. and Crill, W. E., II. Membrane properties of cat spinal motoneurons, Arch. Neurol., 26 (1972) 162-168.

$20 \mathrm{Katz}$, B. and Miledi, R., A study of synaptic transmission in the absence of nerve impulses, $J$. Physiol. (Lond.), 192 (1967) 407-436.

21 Kostyuk, P. G. and Krishtal, O. A., Separation of sodium and calcium currents in the somatic membrane of mollusc neurones, J. Physiol. (Lond.), 270 (1977) 545-568.

22 Llinas, R. and Hess, R., Tetrodotoxin-resistant dendritic spikes in avian Purkinje cells, Proc. nat. Acad. Sci. U.S.A., 73 (1976) 2520-2523.

23 Llinas, R., Steinberg, I., Walton, K., Presynaptic calcium currents and their relation to synaptic transmission: voltage clamp study in squid giant synapse and theoretical model for the calcium gate, Proc. nat. Acad. Sci. U.S.A., 73 (1976) 2918-2922.

24 Llinas, R. and Sugimori, M., Electrophysiological properties of in vitro Purkinje cell dendrites in mammalian cerebellar slices, J. Physiol. (Lond.), 305 (1980) 197-213.

25 Matsuda, Y., Yoshida, S. and Yonezawa, T., A Ca-dependent regenerative response in rodent dorsal root ganglion cells cultured in vitro, Brain Research, 115 (1976) 334-338.

26 Matsuda, Y., Yoshida, S. and Yonezawa, T., Tetrodotoxin sensitivity and Ca component of action potentials of mouse dorsal root ganglion cells cultured in vitro, Brain Research, 154 (1978) 69-82.

27 McAfee, D. A. and Yarowsky, P. J., Calcium-dependent potentials in the mammalian sympathetic neurone, J. Physiol. (Lond.), 290 (1979) 507-523.

28 Molenaar, W. H. and Spector, I., The calcium action potential and a prolonged calcium dependent after-hyperpolarization in mouse neuroblastoma cells, J. Physiol. (Lond.), 292 (1979) 297306.

29 Mudge, A. W., Leeman, S. E. and Fischbach, G. D., Enkephalin inhibits release of substance P from sensory neurons in culture and decreases action potential duration, Proc. nat. Acad. Sci. U.S.A., 76 (1979) 526-530.

30 Pitman, R. M., Intracellular citrate or externally applied tetraethylammonium ions produce calcium-dependent action potentials in an insect motoneurone cell body, J. Physiol. (Lond.), 291 (1979) 327-337.

31 Ransom, B. R. and Holz, R. W., Ionic determinants of excitability in cultured dorsal root ganglion and spinal cord cells, Brain Research, 136 (1977) 445-453.

32 Ransom, B. R., Neale, E., Henkart, M., Bullock, P. N. and Nelson, P. G., Mouse spinal cord in cell culture. I. morphology and intrinic neuronal electrophysiologic properties, J. Neurophysiol., 40 (1977) 1132-1150.

33 Schwartzkroin, P. A. and Slawsky, M., Probable calcium spikes in hippocampal neurons, Brain Research, 135 (1977) 157-161.

34 Schwindt, P. and Crill, W., Role of a persistent inward current in motoneuron bursting during spinal seizures, J. Neurophysiol., 43 (1980) 1296-1318.

35 Weakly, J. N., Effect of barbiturates on 'quantal' synaptic transmission in spinal motoneurons, J. Physiol. (Lond.), 204 (1969) 63-77.

36 Wong, R. K. S. and Prince, D. A., Dendritic mechanisms underlying penicillin induced epileptiform activity, Science, 204 (1979) 1228-1231.

37 Wong, R. K. S., Prince, D. A. and Basbaum, A. I., Intradendritic recordings from hippocampal neurons, Proc. nat. Acad. Sci. U.S.A., 76 (1979) 986-990.

38 Yoshida, S. and Matsuda, Y., Studies on sensory neurons of the mouse with intracellular-recording and horseradish peroxidase-injection techniques, $J$. Neurophysiol., 42 (1979) 1134-1145. 